\title{
The Chronic Care Model and Exercise Discussions during Primary Care Diabetes Encounters
}

\author{
Neela K. Patel, MD, MPH, and Michael L. Parchman, MD, MPH
}

Background: Discussing self-care activities like exercise is challenging with the many competing demands during primary care encounters. Our objective was to study the relationship between the Chronic Care Model (CCM) score in the clinic and time spent by the physicians discussing exercise during encounters with diabetic patients.

Methods: Consecutive patients with diabetes across 20 primary care clinics in South Texas were included. Time spent discussing exercise was determined using the Davis Observation Code on audio recordings of the visits. Clinicians completed the Assessment of Chronic Illness Care survey, a validated measure of the extent to which care delivered is consistent with the CCM. Data were analyzed using hierarchical linear models.

Results: A total of 162 transcribed recordings were analyzed. Age, the number of problems addressed, stage of change (SOC), and overall length of the visit were associated with time spent discussing exercise. There was a significant relationship between clinic CCM score and time spent by physicians advising about exercise, independent of SOC for exercise $(P<.01)$. Also, a discussion about exercise was more likely to occur with patients who were in the contemplation SOC for exercise.

Conclusions: Discussions of exercise may be 18 to 33 seconds longer in clinics with full implementation of the CCM compared with those with basic implementation. Facilitating more complete CCM implementation in clinics with a basic level of CCM that serve a population of patients who are sedentary may realize the most benefit. (J Am Board Fam Med 2011;24:26-32.)

Keywords: Chronic Care Model, Diabetes, Exercise, Patient Education

Primary care physicians can play an important role in supporting self-care activities, such as exercise among patients with a chronic illness. ${ }^{1}$ Despite the

\footnotetext{
This article was externally peer reviewed.

Submitted 15 June 2010; revised 20 September 2010; accepted 4 October 2010.

From the Department of Family and Community Medicine, University of Texas Health Science Center at San Antonio (NKP \& MLP); and Verdict Health Services Research Program, South Texas Veterans Health Care System (MLP), San Antonio.

Funding: The research reported was supported by the Agency for Healthcare Research and Quality (AHRQ K08 HS013008-03) and the Bureau of Health Professions; Health Resources and Services Administration Department of Health and Human Services grant D01 HP08795; The John A Hartford Foundation Center for Excellence in Geriatrics Education; and by the Department of Veterans Affairs.

Conflict of interest: none declared.

Disclaimer: The views expressed in this article are those of the authors and do not necessarily represent the views of the Department of Veterans Affairs.

Corresponding autbor: Neela Patel, MD, MPH, The University of Texas Health Science Center at San Antonio, MC-7795, 7703 Floyd Curl Drive, San Antonio, TX 782293900 (E-mail: Pateln4@uthscsa.edu).
}

evidence of effectiveness of preventive services and supporting self-management behaviors, ${ }^{2,3}$ primary care encounters are bounded by time constraints within which multiple diagnoses, problems, and patient concerns compete with each other for a place on the agenda. ${ }^{4}$ These multiple competing demands often result in health behavior counseling taking a back seat to issues and concerns considered more pressing or important. ${ }^{2}$

The Chronic Care Model (CCM) suggests that interactions between informed activated patients and prepared, proactive care teams can produce better care and improved outcomes. ${ }^{1,5}$ The CCM was developed by identifying 6 evidence-based elements associated with improved chronic illness outcomes. It is based on the concept that when proactive patient care teams provide care to informed, activated patients, clinical quality and outcomes will improve. For example, for patients to engage in proactive care (delivery system design), practices need to be able to view all the patients in 
their panels (clinical information systems) who need certain guideline-based treatments (decision support), and patients must agree to any changes in their care and integrate them into their lives (selfmanagement support). ${ }^{1}$

Considerable experience using the CCM to improve the quality of chronic illness care has accumulated over the past decade. ${ }^{6}$ Evidence suggests that practices redesigned in accordance with the CCM generally improve the quality of care and the outcomes for patients with various chronic illnesses. ${ }^{7,8}$ This finding seems to be consistent in both US and international settings. ${ }^{9,10}$

Of particular interest is evidence suggesting that the CCM is associated with clinical outcomes such as control of glycosylated hemoglobin. This relationship seems to be mediated by patients' self-care activities, such as diet and exercise. ${ }^{5}$ The Transtheoretical Model ${ }^{11,12}$ asserts that there are distinct stages in the adoption of health behavior change, which individuals cycle through en route to consistent adoption of the health behavior in question. In previous studies we found a relationship between the stage of change (SOC) for exercise and the CCM. We found that patients in clinics with high CCM scores were more likely to be in the maintenance SOC for exercise and other self-care behaviors. $^{3,5,7,8,13-17}$ One possible explanation for this relationship is that in clinics with higher CCM scores patients receive more advice and support to improve their level of exercise.

The purpose of this study was to expand our understanding of the relationship between the CCM and exercise SOC. We hypothesize that presence of the CCM in primary care clinics will be associated with the amount of time spent discussing exercise during the visit.

\section{Methods \\ Setting}

The results reported here are from data collected in the Direct Observation of Diabetes Care study. ${ }^{5,13,17}$ Details of the methods have been explained in previous studies. ${ }^{7,8}$ The data were collected as part of an in-depth examination of the of quality of care delivered to patients with type 2 diabetes across a wide variety of primary care settings in 2002 to 2003. The study was cross-sectional and was conducted in 20 primary care clinics with 45 primary care physicians from across South Texas: 11 solo physician clinics (11 physicians); 3 group practice settings (10 physicians); 1 community health center (1 physician); 2 Veterans Affairs primary care clinics (11 physicians); and 3 city/county health clinics for uninsured patients (12 physicians).

\section{Patients and Data Collection}

Within each clinic, 8 to 10 consecutive patients who presented with an established diagnosis of type 2 diabetes were recruited to participate in the study and had their visit audio-recorded. None of the patients who were approached declined participation. After the visit patients completed an exit survey and were asked about their SOC for self-care behaviors for exercise. The SOCs were adopted from the Transtheoretical Model: precontemplation, contemplation, preparation, action, and maintenance. ${ }^{11,12,16}$ In addition to describing specific SOC in the analysis, we also constructed an SOC variable as a dichotomous outcome: yes, the patient is in the maintenance stage of change for the selfcare behavior, or no, the patient is not in the maintenance SOC for the self-care behavior. Patients in the maintenance SOC reported that they have been adherent to the behavior for at least the past 6 months. Additional patient characteristics included in the analysis were age, sex, race/ethnicity, and self-reported health status (excellent, very good, good, fair, poor).

\section{Measurements}

Chronic Care Model

We used the Assessment of Chronic Illness Care survey (ACIC) to determine the degree to which care in each clinic was consistent with the CCM. ${ }^{1}$ This 25-item survey, which measures the presence of the elements of the CCM, was completed by all clinicians in each clinic (physicians, nurse practitioners, and physician assistants). Each item is scored on a 0 to 11 scale and provides subscale scores for each of the 6 CCM components. Several studies support the validity of this instrument. For example, all 6 subscales were responsive to process of care improvement in a study of an intervention for diabetes and congestive heart failure, ${ }^{17-20}$ whereas in a collaborative intervention study overseen by the Institute for Health Care Improvement, ratings by an external team on the depth of implementation of the elements of the CCM were significantly associated with the overall ACIC score for 5 of the 6 elements. $^{6}$ 
Table 1. Descriptive Statistics of Patients and Visits $(\mathrm{n}=162)$

\begin{tabular}{lc}
\hline Variable & \\
\hline Patient characteristics & \\
Age, yr (mean [SD]) & $58.0(13.1)$ \\
Hispanic (\%) & $54(0.0)$ \\
High school graduate (\%) & 72.0 \\
Self-rated health fair or poor (\%) & 44.1 \\
Diagnoses (mean [SD]) & $5.1(2.2)$ \\
Chronic medications (mean [SD]) & $6.7(3.1)$ \\
Visit characteristics & 23.0 \\
Acute visit (\%) & $18.1(8.2)$ \\
Duration of visit, min (mean [SD]) & $20.0(36.0)$ \\
Time discussing exercise, sec (mean [SD]) \\
\hline
\end{tabular}

\section{Content of Encounter (Exercise Discussion)}

Direct observation and audio recordings of the visit allowed a trained coder to discriminate the presence or absence of discrete behaviors that have been operationally defined, systematically minimizing experimenter basis. The Davis Observation Code is a reliable and valid interactional analysis system that has been used to characterize differences in physician practice styles in a variety of previous studies. ${ }^{7,21-23}$ The coder recorded the occurrence or nonoccurrence of each of 20 clinically significant behaviors during successive 15 -second observation intervals of the medical encounter, including a code for the discussion of exercise. For purposes of this analysis, the number of 15 -second intervals devoted to a discussion of exercise were converted into minutes by multiplying by 4 .

\section{Data Analyses}

A mean total CCM score was calculated for each clinic. In addition to characterizing individual SOC, we constructed a SOC variable as a dichotomous outcome for the patient self-care behavior for exercise: the patient is in one of the 5 SOCs (precontemplation, contemplation, preparation, action, or maintenance). Additional patient and visit characteristics such as age, sex, length of visit, reason for visit, maintenance SOC, and SOC for exercise were controlled for in the final model. To account for clustering of patients within clinics, a 2-level hierarchical regression model was used, with patient- and visit-level predictors entered at level 1 and clinic ACIC scores entered at level 2. The number of 15 -second intervals spent discussing exercise was the dependent or outcome vari- able. Patient-level predictors included age, sex, whether or not the visit was an acute visit, whether or not the patient was in the maintenance SOC for exercise, and the length of visit (dependent variable: time spent discussing exercise). We also controlled for precontemplation SOC for exercise in the final model.

\section{Results}

A total of 188 visits were audio recorded, but 26 visits were in Spanish, so 162 visits were coded using the Davis observation codes in the year 2003. Patient and visit characteristics are present in Table 1. The average age of patients was 58 years (range, 35-71 years). More than half (54\%) were Hispanic, consistent with the demographics of the South Texas region. A majority were high-school graduates. Less than half rated their health as fair/poor. In addition to diabetes, the average number of chronic illness diagnoses for each patient was 5, ranging from 2 to 7 comorbid conditions. Patients were taking an average of 7 medications (range, 4-10). The purpose of most visits was for a routine checkup; only $23 \%$ were acute visits. The average duration of a visit was approximately 18 minutes (range, 10-26 minutes). The average time discussing exercise was 22 seconds (range, 8-36 seconds).

Table 2 presents the predictors for the time spent discussing exercise. The number of problems

Table 2. Bivariate Relationships between Each Predictor and Time Spent* Discussing Exercise $(\mathrm{n}=162)$

\begin{tabular}{lc}
\hline & $\begin{array}{c}\text { Length of Time } \\
\text { Discussing Exercise }\end{array}$ \\
\hline Age & \\
$\quad$ Pearson correlation & 0.00 \\
$\quad$ Significance (2-tailed) & 0.96 \\
Number of symptoms/problems & \\
$\quad$ raised by patient & \\
Pearson correlation & 0.08 \\
Significance (2-tailed) & 0.31 \\
Number of problems addressed & \\
$\quad$ during visit & \\
Pearson correlation & 0.26 \\
Significance (2-tailed) & $<0.001$ \\
Length of Visit & \\
Pearson correlation & 0.19 \\
Significance (2-tailed) & 0.015 \\
\hline
\end{tabular}

*Seconds was unit measurement of time spent. 
Table 3. Stage of Change (SOC) of Exercise and If Time Was Spent Discussing Exercise

\begin{tabular}{lcc}
\hline & \multicolumn{2}{c}{$\begin{array}{c}\text { Any Time Spent } \\
\text { Discussing Exercise? }\end{array}$} \\
\cline { 2 - 3 } SOC for Exercise & Yes & No \\
\hline Maintenance $(\mathrm{n}=72)$ & $28(38.9)$ & $44(61.1)$ \\
Action $(\mathrm{n}=18)$ & $9(50.0)$ & $9(50.0)$ \\
Preparation $(\mathrm{n}=35)$ & $16(45.7)$ & $19(54.35)$ \\
Contemplation $(\mathrm{n}=16)$ & $11(68.8)$ & $5(31.3)$ \\
Precontemplation $(\mathrm{n}=21)$ & $2(9.5)$ & $19(90.5)$ \\
Total $(\mathrm{n}=162)$ & $66(40.7)$ & $96(59.3)$ \\
\hline
\end{tabular}

Values provided as $\mathrm{n}(\%)$.

Pearson $\chi^{2}=14.476$; df $=4 ; P=.005$.

addressed during the visit, along with the overall length of the visit, was positively associated with time spent discussing exercise; however, age and the number of symptoms or problems raised by the patient were not. SOC for exercise was also associated with the likelihood that exercise was discussed (Table 3). For patients who were in the contemplation, preparation, and action SOCs, the likelihood of discussing exercise was higher than among those in the maintenance and precontemplation SOCs $(P=.005)$.

In the hierarchical regression model (Table 4), the CCM score was associated with the time spent during the encounter discussing exercise, after adjusting for patient and visit characteristics. For each 1-point increment in the CCM score, the time spent discussing exercise increased by approximately 3 seconds.

\section{Discussion}

In clinics where the CCM is more fully implemented, primary care physicians spend more time discussing exercise during encounters with patients who have type 2 diabetes. This is true even after controlling for the reason for the visit, the length of visit, and the individual patient's exercise SOC. What does this mean in more practical terms? The CCM score ranges from 0 to 11 , with scores from 9 to 11 representing full CCM implementation and scores of 0 to 3 (a range of difference from 6-11 points) representing basic implementation. The regression coefficient suggests that each 1-point increase is associated with an additional 3 seconds of exercise discussion. Therefore, discussion of exercise may be 18 to 33 seconds longer in clinics with full implementation of the CCM compared with those with basic implementation.

Although overall time spent counseling on exercise-related matters was statistically correlated with CCM scores, the time differential was very small and the total range of time spent in this activity was very limited across the entire visit cohort. Behavioral change "counseling" for this brief period of time is similar to other studies in which providers incorporated brief physical activity advice into routine primary care visits with little disruption. The response to physician advice protocol was positive and participation in the study was viewed as beneficial. ${ }^{24,25}$ Primary care settings provide an important opportunity to promote physical activity for adults. Although those most at risk are more likely to receive such advice, there are many more that may benefit. ${ }^{26}$ Evidence-based primary care physical activity counseling protocols can be translated into routine practice. ${ }^{27}$ Opportunistic strategies show promise for a higher yield of identification of patients at risk and leading to advice. ${ }^{28}$

In addition, patient SOC for exercise seems to predict the likelihood that a discussion about exercise will occur. It is less likely to occur with patients who are in the precontemplation and maintenance SOCs and more likely to occur with patients in the contemplation, preparation, and action SOCs. Patient-initiated health behavior discussions are more likely to receive advice if they explicitly indicated readiness to change. A discussion about exercise occurred in only $9 \%$ of encounters with patients who were in the precontemplation SOC, but a discussion about exercise occurred in $69 \%$ of encounters when the patient was in contemplation

Table 4. Multivariable Random Effects Model Predicting Time Spent Discussing Exercise

\begin{tabular}{lccr}
\hline Fixed Effect & Coefficient & SE & $P$ \\
\hline CCM score & 0.21 & 0.06 & $<.01$ \\
Length of time (min) & 0.03 & 0.03 & .36 \\
Age (years) & -0.01 & 0.01 & .22 \\
Female Sex & -0.46 & 0.21 & .04 \\
Acute visit & -0.64 & 0.31 & .05 \\
Maintenance SOC for exercise & -0.27 & 0.23 & .27 \\
Precontemplation SOC for & -1.03 & 0.27 & $<.01$ \\
$\quad$ exercise & & &
\end{tabular}

Dependent variable: number of 15 -second intervals spent discussing exercise. CCM was adjusted for all of the other variables included in the model.

CCM, Chronic Care Model; SOC, stage of change. 
stage (see Tables 2 and 3). It is possible that primary care physicians are aware of the SOC and tailor the time during the encounter to match the SOC for exercise. Amid complex visits with a high level of competing demands, primary care physicians are somehow aware of the SOC of their patients, as reflected in varying percentage of exercise discussions. Future studies will need to explore this finding in more detail.

Our findings are consistent with other studies suggesting that the CCM is related to clinician behavior. For example, implementation or presence of the CCM was associated with traditional process of quality of care indicators dependent on clinician behavior, such as performance of a foot or eye examination for patients with diabetes. ${ }^{17,19,29,30}$ In other studies where CCM scores were higher, providers were more likely to use recommended therapies such as lipid-lowering and angiotensinconverting enzyme inhibition therapy.

Other studies have found a relationship between the CCM and clinical outcomes, such as glycosylated hemoglobin. ${ }^{5,31,32}$ It is possible that the pathway to better clinical outcomes for patients seen in clinics with higher CCM scores is through improved patient self-care behavior such as exercise. For example, in settings with higher CCM scores, patients with asthma were more likely to monitor their peak flows and have a written action plan, and their quality of life improved. ${ }^{33}$

Patient age was found not to be an important predictor of issues addressed during the medical visit. This is contrary to studies that have shown that health promotion discussions, asking patients to make changes in their behavior to increase or promote health, occurred more frequently with younger patients. ${ }^{28,34-38}$ Irrespective of patient age, exercise counseling was significantly more likely to take place with patients who presented with more problems and who were in for a routine visit. Alternatively, physicians may perceive that a discussion of exercise is needed with patients who have more problems and more medications, and that these patients were more suited to discussions of health promotion. Moreover, the competing demands of managing chronic conditions in older patients during the scheduled visit time may preclude addressing other issues such as health promotion. ${ }^{4,13,39}$

Why should the presence of the CCM be related to length of discussion about exercise? It is possible that higher CCM scores reflect a more proactive approach to improving exercise. Perhaps patients seen in clinics with higher CCM scores are more likely to be actively involved in their own care and ask questions related to exercise, thus prompting a discussion of exercise. Also, clinics with higher CCM scores may have clinic structures and processes such as reminders and resources that support clinicians and encourage them to discuss exercise. For example, computer prompts, encounter templates, or a flow chart may increase the time devoted to a discussion of exercise. It is also possible that clinics with a higher CCM score may have longer visits, but in further analysis of the data this did not prove to be true (data not shown). Developed more than a decade ago, the CCM is a widely adopted approach to improving ambulatory care that has guided clinical quality initiatives in the United States and around the world. Here we examined the evidence of the CCM's effectiveness by reviewing articles published since 2000 that used one of 5 key CCM articles as a reference. Accumulated evidence seems to support the CCM as an integrated framework to guide practice redesign. Although work remains to be done in areas like cost-effectiveness, these studies suggest that redesigning care using the CCM leads to improved patient care and better health outcomes. ${ }^{6}$

One limitation of this study is the cross-sectional nature of the data. For example, it is possible that some physician characteristics would result in both the presence of the CCM and a longer discussion of exercise. Another limitation of this study is an inability to draw any conclusion or causality or the direction of observed relationships because of the cross-sectional nature of the data. We do not know whether the SOC was influencing the time spent discussing exercise or if visit time spent discussing exercise was influencing exercise SOC, as reported after the visit by the patients on the administered survey. It is possible that some as-yet unrecognized factor may influence both the CCM score and the reason for increased time spent by physicians advising about exercise. Prospective studies are needed to further evaluate the effect of CCM on the time spent discussing exercise and whether this time equates to patient behavior change. 


\section{Conclusions}

In primary care clinics where care is more consistent with the CCM, more time is spent discussing exercise during a routine visit encounter. These clinics may have prepared proactive teams interacting with informed, activated patients, resulting in better support for self-care behaviors. Self-management programs for chronic diseases such as diabetes, hypertension, and heart disease probably produce clinically important benefits. Because primary care clinics are the principal source of the tools for self-care and because they support patients who need to learn to manage complex chronic diseases, a greater focus on strengthening the presence of the CCM may be needed in these settings.

\section{References}

1. Bonomi AE, Wagner EH, Glasgow RE, VonKorff M. Assessment of chronic illness care (ACIC): a practical tool to measure quality improvement. Health Serv Res 2002;37:791-820.

2. Yarnall KS, Pollak KI, Ostbye T, Krause KM, Michener JL. Primary care: is there enough time for prevention? Am J Public Health 2003;93:635-41.

3. Parchman ML, Flannagan D, Ferrer RL, Matamoras M. Communication competence, self-care behaviors and glucose control in patients with type 2 diabetes. Patient Educ Couns 2009;77:55-9.

4. Parchman ML, Pugh JA, Romero RL, Bowers KW. Competing demands or clinical inertia: the case of elevated glycosylated hemoglobin. Ann Fam Med 2007;5:196-201.

5. Parchman ML, Pugh JA, Wang CP, Romero RL. Glucose control, self-care behaviors, and the presence of the chronic care model in primary care clinics. Diabetes Care 2007;30:2849-54.

6. Coleman K, Austin BT, Brach C, Wagner EH. Evidence on the chronic care model in the new millennium. Health Aff (Millwood) 2009;28:75-85.

7. Kaissi AA, Parchman M. Assessing chronic illness care for diabetes in primary care clinics. Jt Comm J Qual Patient Saf 2006;32:318-23.

8. Kaissi AA, Parchman M. Organizational factors associated with self-management behaviors in diabetes primary care clinics. Diabetes Educ 2009;35:843-50.

9. Sunaert P, Bastiaens H, Feyen L, et al. Implementation of a program for type 2 diabetes based on the chronic care model in a hospital-centered health care system: "the Belgian experience." BMC Health Serv Res 2009;9:152.

10. Wagner EH, Austin BT, Davis C, Hindmarsh M, Schaefer J, Bonomi A. Improving chronic illness care: translating evidence into action. Health Aff (Millwood) 2001;20:64-78.
11. Prochaska JO, DiClemente CC, Norcross JC. In search of how people change. applications to addictive behaviors. Am Psychol 1992;47:1102-14.

12. Prochaska JO, Velicer WF, Rossi JS, et al. Stages of change and decisional balance for 12 problem behaviors. Health Psychol 1994;13:39-46.

13. Parchman ML, Romero RL, Pugh JA. Encounters by patients with type 2 diabetes-complex and demanding: an observational study. Ann Fam Med 2006;4:40-5.

14. Parchman ML, Noel PH, Lee S. Primary care attributes, health care system hassles, and chronic illness. Med Care 2005;43:1123-9.

15. Parchman ML, Burge SK. The patient-physician relationship, primary care attributes, and preventive services. Fam Med 2004;36:22-7.

16. Parchman ML, Arambula-Solomon TG, Noel PH, Larme AC, Pugh JA. Stage of change advancement for diabetes self-management behaviors and glucose control. Diabetes Educ 2003;29:128-34.

17. Parchman M, Kaissi AA. Are elements of the chronic care model associated with cardiovascular risk factor control in type 2 diabetes? Jt Comm J Qual Patient Saf 2009;35:133-8.

18. Pearson ML, Wu S, Schaefer J, et al. Assessing the implementation of the chronic care model in quality improvement collaboratives. Health Serv Res 2005; 40:978-96.

19. Solberg LI, Crain AL, Sperl-Hillen JM, Hroscikoski MC, Engebretson KI, O'Connor PJ. Care quality and implementation of the chronic care model: a quantitative study. Ann Fam Med 2006;4:310-6.

20. Sperl-Hillen JM, Solberg LI, Hroscikoski MC, Crain AL, Engebretson KI, O'Connor PJ. Do all components of the chronic care model contribute equally to quality improvement? Jt Comm J Qual Saf 2004;30:303-9.

21. Ackermann RT, Thompson TJ, Selby JV, et al. Is the number of documented diabetes process-of-care indicators associated with cardiometabolic risk factor levels, patient satisfaction, or self-rated quality of diabetes care? The Translating Research into Action for Diabetes (TRIAD) study. Diabetes Care 2006; 29:2108-13.

22. Bertakis KD, Callahan EJ. A comparison of initial and established patient encounters using the davis observation code. Fam Med 1992;24:307-11.

23. Callahan EJ, Bertakis KD. Development and validation of the davis observation code. Fam Med 1991; 23:19-24.

24. Albright CL, Cohen S, Gibbons L, et al. Incorporating physical activity advice into primary care: physician-delivered advice within the activity counseling trial. Am J Prev Med 2000;18:225-34.

25. Anderson RT, King A, Stewart AL, Camacho F, Rejeski WJ. Physical activity counseling in primary care and patient well-being: do patients benefit? Ann Behav Med 2005;30:146-54. 
26. Berger BA, Hudmon KS. Readiness for change: implications for patient care. J Am Pharm Assoc (Wash) 1997;NS37:321-9.

27. Eakin EG, Brown WJ, Marshall AL, Mummery K, Larsen E. Physical activity promotion in primary care: bridging the gap between research and practice. Am J Prev Med 2004;27:297-303.

28. Flocke SA, Kelly R, Highland J. Initiation of health behavior discussions during primary care outpatient visits. Patient Educ Couns 2009;75:214-9.

29. Solberg LI, Asche SE, Pawlson LG, Scholle SH, Shih SC. Practice systems are associated with highquality care for diabetes. Am J Manag Care 2008;14: 85-92.

30. Strickland PA, Hudson SV, Piasecki A, et al. Features of the Chronic Care Model (CCM) associated with behavioral counseling and diabetes care in community primary care. J Am Board Fam Med 2010; 23:295-305.

31. Feifer C, Ornstein SM, Nietert PJ, Jenkins RG. System supports for chronic illness care and their relationship to clinical outcomes. Top Health Inf Manage 2001;22:65-72.

32. Parchman ML, Pugh JA, Noel PH, Larme AC. Continuity of care, self-management behaviors, and glucose control in patients with type 2 diabetes. Med Care 2002;40:137-44.
33. Tsai AC, Morton SC, Mangione CM, Keeler EB. A meta-analysis of interventions to improve care for chronic illnesses. Am J Manag Care 2005;11: 478-88.

34. Croteau K, Schofield G, McLean G. Physical activity advice in the primary care setting: Results of a population study in new zealand. Aust $\mathrm{N}$ Z J Public Health 2006;30:262-7.

35. Eakin E, Brown W, Schofield G, Mummery K, Reeves M. General practitioner advice on physical activity-who gets it? Am J Health Promot 2007;21: 225-8.

36. Flocke SA, Stange KC, Goodwin MA. Patient and visit characteristics associated with opportunistic preventive services delivery. J Fam Pract 1998;47: 202-8.

37. Glasgow RE, Eakin EG, Fisher EB, Bacak SJ, Brownson RC. Physician advice and support for physical activity: results from a national survey. Am J Prev Med 2001;21:189-96.

38. Kreuter MW, Scharff DP, Brennan LK, Lukwago SN. Physician recommendations for diet and physical activity: which patients get advised to change? Prev Med 1997;26:825-33.

39. Presseau J, Sniehotta FF, Francis JJ, Campbell NC. Multiple goals and time constraints: perceived impact on physicians' performance of evidence-based behaviours. Implement Sci 2009;4:77. 